\title{
Gen 1, 26-27 e Gen 2, 7 nella interpretazione patristica (le scuole "asiatica" e "alessandrina")
}

La dottrina dell'uomo "immagine di Dio" è il nucleo dell' antropologia biblica e patristica. I Padri non hanno tra le loro mani il testo in lingua ebraica con i vocaboli selem e, in posizione subordinata, demuth (quindi: "immagine somigliante"), ma il testo nella traduzione greca dei LXX, a sua volta poi tradotto in latino, che suona cosi: immagine e somiglianza. L'osservazione vale sia pez il primo racconto della creazione (Gen 1, 26-27; 5, 3; 9, 6; Sap 2, 23; Sir 17, 3) che per il secondo (Gen 2, 7). Si ricordi inoltre che 1'A.T. viene letto alla luce del N.T. e quindi ai testi veterotestamentari si devono accostare i testi paolini di Col 1,15 e 2 Cor 4, 4 senza omettere 1 Cor 15, 45-49; 1 Cor 11, 7; 2 Cor 3, 18 e Rom 8, 29, nonché Fil 3, 21) (1).

I Padri si domandano: Dove si colloca l'immagine di Dio nell'uomo? Nell'anima e nel corpo, o nella sola anima? Siamo al cuore dell' antropologia patristica. Per una risposta ascoltiamo la voce delle due "scuole".

\section{1) LA SCUOLA ASIATICA}

È necessario ricordare che alla base sta la polemica antignostica, tesa a riscattare la carne dall'ipoteca platonica e gnostica (2), secondo cui "ciò che è materiale non è capace di salvezza" (detto gnostico affiorante dovunque in Ireneo e Tertulliano) (3). Il motivo anti-gnostico e la collocazione dell'immagine nella "carne" è dottrina unica in questa tradizione.

Lo Ps.-Giustino, nel De resurrectione:

(1) Per una bibliografía essenziale vedi: G. KITTEL, s.v. Eikòn, in Th. W. N.T., 11, 394-395.

(2) Per 1'attualità del tema vedi: E. Samek Lodovici, Metamorfosi della Gnosi Quadri della dissoluzione contemporanea, Milano 19912; L. Bouyer - L. Dattrino, La spiritualità dei Padri, Bologna 1984 (Storia della Spiritualità 3/A), p. 123 ss.

(3) Qualche citazione da Ireneo: "Animae autem eorum soli esse salutem, corpus enim natura corruptibile" (Adv. Haer, 1, 19, 3: Basilide); "Salutem autem solum animarum esse futuram, earum quae eius doctrinam didicissent, corpus autem. videlicet quoniam a terra sit sumptum, impossibile esse participare salutem" (Adv. Haer. 1, 25, 2: Marcione). 
"Annon enim dicit Scriptura: "Faciamus hominem ad imaginem et similitudinem nostram?". Carneum videlicet hominem dicit (sarkikón ánthropon). Ait enim Scriptura: "Et accepit Deus pulverem de terra et formavit hominem". Perspicuum igitur est hominem, qui formatus est ad imaginem Dei, fuisse carnem. Ad haec quomodo non absurdum sit dicere carnem, quae a Deo ad imaginem ipsius facta est, vilem esse ac nihili?" (De res., 6; PG 6, 1583-1584).

Teofilo A., commentando la creazione dell'uomo, afferma che plasmazione (plasis) e immagine di Dio si richiamano l'un l'altra: "Il cielo è opera di lui, la terra è sua fattura, il mare sua creazione, l'uomo plasma e immagine sua (plasma kai eikón autoú)" (Ad Aut. 1, 4). L'uomo che è al centro della creazione non è l'anthropos ideale, svincolato dal mondo sensibile, ma l'uomo nella sua natura corporea. È quest'uomo che è creato "a immagine e somiglianza di Dio". Nella sua polemica contro gli asiatici, Origene fa espresso riferimento a Melitone, di cui cita l'opera da lui contestata. La notizia di Origene è preziosa in quanto attesta nel vescovo di Sardi 1'identità del plasma e dell'eikón. Origene, condizionato dalla sua concezione dottrinale in genere, e dalla polemica contro l'esegesi ingenua che di Gen 1, 26 davano gli antropomorfiti presi di mira da Celso (C. Celsum VI, 62 s. (4), non poteva accettare l'ipotesi dell'immagine di Dio riferita al corpo di Adamo, e così non è stato in grado di cogliere 1'esegesi profonda diffusa tra presbiteri dell'Asia, e continuata da Teofilo, Ireneo, Tertulliano e altri. L'interesse di Melitone per l'antropologia appare da altri titoli trasmessi da Eusebio (Storia eccl. IV, 26), come: "Sul plasma" (Peri plásmatos), o "Sull'anima e sul corpo, ovvero dell'unità". A ragione si può affermare che Melitone è un anello importante di quella teologia asiatica per la quale il plasma è immagine di Dio. E sotto questo profilo egli è una delle fonti di Ireneo. Ireneo difende innanzitutto l'unità dell'uomo. L'unità che egli rivendica in Dio, insieme creatore del mondo e Padre del Verbo, la rivendica anche, contro gli stessi avversari, all'interno del composto umano. Egli "si richiama alla costituzione tripartita dell'uomo: corpo, anima, Spirito, come proponeva $I$ Tess 5, 23, che del resto era grosso modo ammessa anche dagli gnostici. Ma si separa da questi in quanto professa che, sotto l'angolo soteriologico, l'uomo completo non si riduce a nessuno di questi costitutivi, separato dagli altri, e in particolare non si limita all'anima o allo spirito: anima e spirito, come anche il corpo, presi isolatamente, non sono che parti dell'uomo totale, il quale si definisce dall'unione e dall'insieme dei tre elementi. È l'uomo totale l'oggetto della salvezza voluta da Dio, il che implica che anche il corpo sia salvato dopo la risurrezione" (5). Non tre uomini, dunque, ma un solo uomo. Testo fondamentale in Adv. haer. V, 6, 1

Anzi, di fronte al predominio eccessivo del nous o pneuma, e al pessimismo ontologico nei riguardi del corpo come fonte del male, Ireneo afferma vigorosamente il primato del corpo: l'uomo è principalmente sarx, plasma. Alla visione discen-

(4) Cfr. L Dattrino, Patrologia, Roma $1982^{2}$ Per avere un' idea del rapporto tra filosofia pagana ed il cristianesimo vedi Dattrino (a cura di), Origene Apologia del cristianesimo,, Padova 1987 (Pagine antologiche da Contra Celsum). J. PÉPIN, Idées grecques sur l'homme et sur Dieu. Paris 1971, p. 168. 
dente, propria degli gnostici (e del pensiero platonico in genere), secondo cui il corporeo sta al termine di una caduta e l'uomo è uno spirito che scopre la sua prigione e la sua degradazione nel vestito di carne, Ireneo oppone la visione ascendente e progressiva della carne fino alle altezze di Dio. La corporeità non si spiega come decadenza, bensi come inizio modesto proiettato nella teleiosis e destinato al vertice dell'increato. "La confutazione dell'errore gnostico (che negava la salvezza del corpo) offre al vescovo di Lione l'occasione di sviluppare tutto il suo pensiero sul posto del corpo nell'economia generale del cristianesimo. Questo primo monumento della teologia del corpo altri lo completeranno, all'occasione lo rettificheranno; ma le sue linee maestre restano. Per Ireneo la condanna del corpo implica il misconoscimento del mistero cristiano: creazione, incarnazione, redenzione" (6). C'è chi ha parlato, a proposito di Ireneo, di "sarcologia", o teologia della carne. È infatti, la carne, immagine della carne di Cristo. Ireneo, come del resto gli esponenti della teologia asiatica, non solo ignora lo schema della "doppia creazione", ma, interpretando i due racconti della Genesi in senso complementare, mette l'accento proprio sulla "carne", sul plasma. Certo, per lui, che si attiene a Tess 5, 23, l'uomo risulta di tre elementi. Ma dei tre: plasma, anima e Spirito, quello che funge da polo di attrazione è la carne, capace di accogliere lo Spirito e di diventare "gloriosa". La vera posta in gioco è la carne.

L'alta concezione che Ireneo ha della carne sembra mettere in ombra la realtà dell'anima. Testi evidenti ne affermano la necessità nel composto umano; tuttavia non si sfugge all'impressione che essa appaia piuttosto in funzione subalterna, come "strumentalizzata" a beneficio della carne. A spiegare lo squilibrio bisogna pensare, più che alle controversie sull'anima nei secc. II e III, alle esigenze polemiche anti-gnostiche che spostarono appunto il dibattito sul punto centrale della salvezza della carne (7).

Con l'accento sul corpo-plasma, resta decisamente affermata l'importanza della carne come componente essenziale, se non preminente, della salvezza. Tale accento, che può sembrare eccessivo, e probabilmente lo è a causa della polemica che lo ha suscitato, rappresenta tuttavia l'approccio più semitico fatto al testo di Gen 1, 26-27. L'enfasi sulla totalità dell'uomo come persona fatta a immagine e somiglianza di Dio, oltre ad essere la caratteristica della teologia asiatica, è quella più vicina al dato biblico.

(6) D. GORCE. s.v. Corps, in Dict. Spirit. II, 2, Paris 1953, coI. 2346. Cfr. pure P.C. Bori, Faccio muove tutte le cose, Roma 1968, PP. 79-80.

(7) Cfr. L. Dattrino, s.v. Ireneo, in Dizionario di Mistica, 140-150. Cfr. A. ORBE, Antropología de San Ireneo, Madrid 1969, passim. Riporto uno dei testi di Tertulliano in cui si rivendica la dignità e la totalità della carne nel processo della salvezza: "La carne è il cardine della salvezza, a tal punto che, quando per mezzo della salvezza l'anima è legata a Dio, è proprio la carne a fare in modo che l'anima possa essere scelta da Dio. Ed ancora, è la carne che viene lavata perché si purifichi l'anima, è la carne che viene unta perché 1'anima sia consacrata, è sulla carne che si fa il segno, perché l'anima sia difesa, è la carne che viene adombrata dalla imposizione delle mani, perché poi anche l'anima sia illuminata dallo Spirito, è la carne che si ciba del corpo e del sangue di Cristo, perché anche l'anima possa essere nutrita di Dio. Non si può dunque separare nella ricompensa coloro che sono congiunti salle opere" (De res. 8, 2-3: da Opere scelte di Q.S.F. Tertulliano, a cura di C. Moreschini, Torino 1974, pp. 789-790). 


\section{2) LA SCUOLA ALESSANDRINA}

L'uomo a immagine non è l'uomo integrale, tanto meno il corpo o la carne, come per la tradizione asiatica, ma l'anima, o meglio il nous, o mens, sede della conoscenza, della libertà e di ogni virtù. Solo indirettamente la sublimità dell'immagine si ripercuote sul corpo, il quale, non potendo partecipare alla natura invisibile e spirituale dell'immagine, si situa a un livello nettamente inferiore. In sostanza, l'uomo è immagine, non nel senso biblico di Gen 1, 26-27, o nel senso paolino che raccorda strettamente $\operatorname{Col} 1$, 15, con Gen 1, 26-27 e con 1 Cor 15, 4549, ossia dell'uomo come totalità, ma secondo la concezione filosofica greca.

Clemente A., che non ammette l'uomo ideale di Filone e che considera l'uomo composto essenzialmente di anima e di corpo, attribuendone a Dio la creazione diretta e immediata, afferma tuttavia che "l'espressione "ad immagine e somiglianza" non si riferisce al corpo, perché è inammissibile che il mortale rassomigli all'immortale, ma all'intelletto e alla ragione, ossia a quelle parti dell'uomo in cui il Signore può fissare convenientemente, come un sigillo, la rassomiglianza rispetto al beneficare e al comandare" (Strom. II, 19, 102; cfr. VI, 9, 72; IV, 22, 139). Dall'ultima frase della citazione si evince che Clemente fa consistere la somiglianza fra uomo e Dio non nell'essere, o essenza, né nella forma, né in qualche qualità (cfr. Strom. II, 16, 74-75), ma nell'agire: kat'enérgheian, ossia in quanto l'uomo opera il bene ed esercita il dominio sopra le cose (8).

Ad acclimatare in campo cristiano la concezione alessandrina fu soprattutto Origene. Allo scopo egli adotta lo schema filoniano della "doppia creazione", secondo cui solo l'uomo "fatto" è ad immagine, non l'uomo "plasmato". A conferma, adduce la categoria paolina dell "uomo interiore" e dell "uomo esteriore". Origene non rigetta il corpo come se fosse fonte di ogni corruzione e male, ma sottolinea la dignità dell' "uomo a immagine" nell'incorporeità dell'anima, più precisamente del nous: l'intelletto solo, infatti, può conoscere Dio e assimilarsi all'oggetto conosciuto.

Diamo un testo sulla "duplice creazione":

"Quest'uomo che (la Scrittura) dice "fatto ad immagine di Dio", non l'identifichiamo con (l'uomo) corporale. Non la figura corporale ha (in sé) l'immagine di Dio; d'altro canto, dell'uomo corporale non si dice che è stato fatto, bensi (si dice che è stato ) plasmato, come è scritto in quel che segue. Dice infatti: “e Dio plasmò 1'uomo" (Gen 2, 7), cioè lo modellò, "dal fango della terra" Quello che è stato fatto ad immagine di Dio,è il nostro uomo interiore, invisibile, incorporale, incorrotto e immortale. In questi aspetti, a maggior ragione, si coglie l'immagine di Dio. Se qualcuno ritiene che sia questo uomo materiale ("corporeum") quello che è stato fatto "a immagine e somiglianza di Dio", è come se ammettesse che persino Dio ha corpo e figura umana. Credere questo di Dio è cosa assolutamente empia" (Hom. in Gen I, 13).

(8) Cfr. B. MONDIN, Antropologia teologica. Alba 1977, p. 93. 
Seguendo Filone e Origene, Gregorio Nisseno ripropone lo schema delle due creazioni: quella dell'immagine di Dio ideale, e quella dell'immagine reale. L'immagine ideale riguarda l'umanità tutt'intera, e si subbietta principalmente sull'anima. Sembra tuttavia che Gregorio non consideri 1'uomo ideale allo stesso modo di Filone (come una realtà creata fin dagli inizi della storia per servire da modello nella produzione dei singoli individui), ma piuttosto come un progetto, un programma ideale escogitato da Dio per l'umanità, ma che storicamente non è mai esistito. È il progetto, il modello a cui l'uomo si deve costantemente ispirare se vuole rassomigliare veramente a Dio. L'immagine è, in realtà, anche il traguardo cui l'uomo giungerà dopo la vita presente, se ha vissuto in conformità ai precetti divini e all'esempio di Cristo.

Gregorio rileva tratti di rassomiglianza indiretta con Dio anche nel corpo (posizione verticale, la mano, i sensi, il volto), in quanto riflesso e irradiazione della bellezza dell'anima, e in quanto strumento per l'attività fisica e intellettuale e per l'esercizio della regalità della medesima. L' immagine reale, storica, fu posta in atto da Dio quando decise di creare l'uomo sessuato, rendendolo cosi simile agli animali. Nell'immagine concreta la somiglianza opera dunque in due direzioni: in direzione di Dio, mediante l'anima e le sue facoltà; in direzione degli animali, mediante il corpo e la sua sessualità:

"L'uomo si trova in mezzo, tra due realtà estremamente lontane tra loro: tra la natura divina che non possiede la corporeità, e la natura animale priva di ragione ... Della natura divina, che è esente dalla distinzione dei sessi, l'uomo deriva il potere della ragione e dell'intelligenza; invece dalla natura animale, priva di ragione, egli trae la struttura del corpo e la distinzione dei sessi" (De hominis opificio, 181 c) (9).

Cirillo A. nega categoricamente, contro gli antropomorfiti, che 1'uomo possa rispecchiare l'immagine di Dio nel suo corpo materiale, e insiste nel dire che l'immagine è solo nell' anima (10).

Tutti i Padri greci, concordemente, ripongono l'immagine nell'anima (11).

(9) La dottrina della sessualità, accanto all'altro punto della distinzione tra l'uomo ideale e quello reale, costituiscono i lati oscuri e discutibili dell'antropologia di Gregorio. Secondo lui, la sessualità è una delle cause principali dell'oscuramento dell'immagine di Dio; essa svolge questo ruolo negativo in quanto è strettamente legata al corpo ed è fonte di molte passioni. Tuttavia egli assegna alla sessualità anche un ruolo positivo. Dopo il peccato originale Dio se ne serve per assicurare la propagazione dell'umanità. Si tratta però sempre di una qualità provvisoria, destinata a scomparire nel regno dei cieli: cfr. B. MONDIN, Antropologia teologica, Alba 1977, p. 98.

(10) Cfr G. A MALONEY,Man the Divine Icon, Pecos, New Mexico 1973, pp. 164-165. Cirillo sottolinea, come del resto tutti gli altri, la peculiarità della creazione dell'uomo: non con la semplice parola, come il resto delle creature, ma con la deliberazione e con l'opera delle mani. L'uomo sulla terra è l'impronta della gloria suprema e immagine della regalità divina sulla terra (Glaphyra in Gen 1, 5).

(11) Attiro l'attenzione sulle omelie X-XI, attribuite a BASILIO, sull'origine dell'uomo: molto in evidenza lo schema della doppia creazione, l'uomo "fatto" e l'uomo "plasmato". Per l'attribuzione, cfr. BASILIO DI CESAREA, Opere ascetiche, a cura di U. NERI, Torino 1980, p. 55 , nota 3 
In Occidente troviamo le stesse posizioni. Ilario, uno degli autori nei quali la diversità delle fonti di ispirazione è più rilevabile (12), presenta una riflessione asistematica, sufficiente tuttavia a darci un quadro del suo pensiero. Parlando della posizione preminente e centrale dell'uomo rispetto a tutte le altre creature, un tema ricorrente nella patristica, Ilario, ispirandosi prevalentemente alla tradizione occidentale, mette in risalto la cura particolare con la quale Dio crea l'uomo: consulto divino, le due mani, ecc.:

"Ob id magnum quiddam est homo" (Tract. in Ps. 118, 2 5., PL IX, 564 c-565 c). Nel Commento a Matteo, una delle più antiche opere esegetiche scritte in latino, in un testo piuttosto difficile (Com. Mt 5, 8; PL IX, 946 b-947 a), Ilario presenta una visione antropologica ispirata a presupposti nettamente diversi da quelli che saranno alla base del suo Commento ai Salmi, presupposti di tipo "asiatico" che estendono al corpo il concetto di immagine e somiglianza con Dio. Evidente sembra l'influsso di Tertulliano, per il quale "la corporeità costituisce la suprema categoria della realtà" (13) Sempre nel Com. Mat., Ilario afferma che il corpo dell'uomo è rivalutato e reso nobile dall'incarnazione di Cristo (Com. Mt., 16, 5; PL IX, 1009 a). Siamo al periodo, prima dell'esilio, in cui Ilario ragiona in maniera autonoma e calca la mano sulla "carne" dell'uomo come sull'elemento piu caratteristico, centro di gravità per l'economia della salvezza (14).

Il successivo contatto con la speculazione orientale lo porta a rivolgere l'attenzione in modo particolare all'anima-spirito, in contrapposizione al corpo-materia. A partire dall'esilio si ha pertanto una spiritualizzazione marcata del concetto di immagine. In proposito, Ilario assume lo schema della "doppia creazione", ossia di una duplice composizione dell'uomo, avvenuta in due momenti distinti: l'anima "fatta" al principio, il corpo "plasmato" in seguito (15). Ilario pone l'immagine nell' anima come se essa sola costituisse l'essenza dell'uomo. Il testo maggiore è in Tr. in Ps. 118 (PL IX, 566 b), dove l'influsso di Origene è rilevabile anche letterariamente (16).

Girolamo, appoggiandosi a Sal 119, 73, come Ilario e forse in dipendenza da lui, distingue, nella creazione dell'uomo, factura e plasmatio; cosi infatti scrive, commentando Ef 2, 10: "ipsius enim sumus factura, creati in Christo lesu ...":

(12) Negli scritti anteriori all'esilio, per es nel Commento a Matteo, Ilario è sotto l'ispirazione degli autori latini, in particolare di Tertulliano e di Cipriano. Negli scritti posteriori all'esilio ( $D e$ Trinitate, Tractatus in Psalmos) è evidente l'influsso di Origene e del pensiero greco in genere. Ciò tuttavia non significa cesura netta tra i due periodi: anche nel le opere posteriori all'esilio ricompaiono tematiche proprie della precedente teologia latina.

(13) A. FlERRO, Sobre la gloria en san Hilario, Roma 1964, p. 24. Cfr. J. DOIGNON, Hilaire de Poitiers avant l'exile, Paris 1971; C. KANNENGIESSER, Hilaire, in "Dict. Spirit.", VIIII, PP. 466-499.

(14) A ORBE, Antropología, p. 13.

(15) Cfr. Tract. in ps.118, PL IX, 565 c-566 b. A differenza di Origene, nel quale la doppia creazione deriva da una visione filosolico-cosmogonica. Ilario sembra dedurre la distinzione dall'analisi del doppio racconto della creazione.

(16) Cfr. A ORBE, Antropología, pp. 11-13. 
"Et diligenter observa, quia non dixerit, ipsius figuratio sumus atque plasmatio: sed, ipsius factura sumus. Plasmatio quippe originem de terrae limo trahit: factura vero iuxta similitudinem et imaginem Dei sumpsit exordium. Quod in Ps. 118, 73 simul positum diversa significat: Manus tuae fecerunt me, el plasmaverunt me. Factura primum locum tenet; deinde plasmatio" (In ep. Ad Eph. 2, 10; PL 26, 470).

Ambrogio ha le sue fonti quasi esclusivamente nel mondo orientale (Filone, Plotino, Origene, Atanasio, Basilio, e altri), anche se egli sa fondere l'insegnamento altrui al calore del suo pensiero di pastore e di guida spirituale. II suo dualismo è riscattato dalla fede e da quell'ottimismo di fondo che ispira un' antropologia complessivamente serena. Ecco i temi ambrosiani (17).

La doppia creazione. Ambrogio parla di "gemina generatio", "gemina substantia", "duo creaturae", "duo homines", dei quali l'uno "factus", l'altro "figuratus"; solo il primo è "ad imaginem":

"Scriptura nos edocet prius hominem ad imaginem esse formatum, postea de luto factum. Sic est ergo homo geminae substantiae, alia est enim facti substantia, alia figurati; ista de Dei Spiritu, illa de limo" (In ps. 118, 10, 18).

"Aliud quod figuratum est, aliud quod factum est" (ivi, 10, 17). “...Geminam hominis generationem expressam: unam secundum imaginem Dei, alteram secundum figmentum de luto terrae" (De Noe et arca, 24, 86) (18).

Differenza tra $i$ due uomini. L'uomo "fatto", ossia 1'anima, è l'essenza dell'uomo; 1'uomo "plasmato", o il corpo, non è elemento essenziale di noi, ma piuttosto un nostro possesso, o meglio un possesso dell' anima:

"Anima igitur nostra ad imaginem Dei est, in hac totus es, quia sine hac nihil es, sed es terra et in terram resolveris" (Exam. 6, 7, 43; In ps. 118, 10, 18).

"Cognosce teipsum, o homo ... in qua (anima) totus est, in qua melior tui portio est" (Exam. 6, 6, 39; 6, 7, 43; In ps. 118, 10, 10...).

Il corpo è per l'anima "hospitium", "terrenum habitaculum", "vestimentum", “carcer", "ergastulum", e simili:

"Non igitur caro tu es. Quid est enim caro sine animae gubernaculo, mentis vigore? Caro hodie sumitur, cras deponitur. Caro temporalis, anima diuturna. Caro amictus est animae, quac se induit quodam corporis vestimento. Non igitur tu vestimentum es, sed qui vestimento uteris" (Exam. 6,7,43; In ps. $118,10,18 ; A b r .2,2,5 \ldots)$.

"Nos mens ... anima et mens" (Exam. 6, 7, 42).

(17) Per l'antropologia di Ambrogio fondamentale è l'opera di W. SEIBEL, Fleisch und Geist beim heiligen Ambrosius, München 1958.

(18) Si legga quest'altro testo: "Sic denique et in Genesi duas tibi creaturas hominis ostendit (...) duo homines, ita et gemina conversatio est, una interioris hominis, altera exterioris" (Ep. 72, 19). 
Ambrogio accenna anche al tema delle due mani, a proposito del Sal 119, 73, e e le identifica, alla stregua della teologia asiatica, con il Cristo e lo Spirito Santo: "Istae sunt manus quae hominem praverunt: Christus et Spiritus" (In ps. 118, 10, 17). Le mani di Dio non plasmarono né le bestie, né gli angeli, ma l'uomo: "Mc autem, inquit, fecisti, me tuis manibus figurasti" (ivi, 10, 25). I1 cielo invece è creato con una sola mano (ivi, 10, 12).

Solo l'anima ad immagine. Il capitolo VIII dell'Esamerone è dedicato a spiegare che l'immagine non è nel corpo, ma nell'anima, meglio nel nous:

"Haec (= anima) est ad imaginem Dei, corpus autem ad speciem bestiarum" (Exam. 6, 7, 43; In ps. 118, lo, 18).

"Ad imaginem autem interior est homo, non iste exterior; ille qui sensu aestimatur, non oculis comprehenditur" (Inst. Virg. 3, 20; In ps. 118, 10, 7).

"Quod enim secundum imaginem est, non est in corpore, sed in anima rationali" (In ps. 118, 10, 15).

Ambrogio, in dipendenza da Basilio, ha pagine vibranti sulla bellezza del corpo (cfr. Exam. 9, 54-74), ma non dice esplicitamente che questa bellezza è segno dell'immagine di Dio.

Agostino, nota il Solignac, desume da Ambrogio il punto fondamentale della sua dottrina, sul quale non cambierà mai: e cioè che l'uomo è fatto a immagine di Dio secondo l'anima, e persino ciò che nell'anima è più elevato, la mens o intellectus, u quale è "ciò che eccelle in essa", è "come il suo volto, il suo occhio interiore e intelligibile”. Questa tesi capitale permette ad Agostino di eliminare l'obiezione manichea che attribuiva ai cristiani la corporeità in Dio, colloca l'immagine in ciò che distingue l'uomo dalle creature inferiori e fonda il suo potere su di esse, ma soprattutto stabilisce un rapporto immediato tra l'uomo e Dio (nulla interposita creatura) (19). Qualche testo:

"Non secundum formam corporis homo factus est ad imaginem Dei, sed secundum rationalem mentem. Cogitatio quippe turpiter vana est, quae opinatur Deum membrorum corporalium lineamentis circumscribi atque finiri ... Nulli dubium est non secundum corpus, neque secundum quamlibet animi partem, sed secundum rationalem mentem, ubi potest esse agnitio Dei, hominem factum ad imaginem eius qui creavit eum" (De Trinit. 12, 7, 12).

"Fecit et hominem ad imaginem et similitudinem suam in mente: ibi est enim imago Dei” (De symb. 1, 2).

Nella mente Agostino distingue ulteriormente due rationes: quella inferior, volta alle cose del mondo, e la superior, rivolta verso le verità eteme, e quindi verso

(19) De Trin. II, 5, 8. Cfr. A SOLIGNAC, Image dans la patristique latine, col. 1418. Secondo il Courcelle, Agostino sarebbe stato vivamente colpito dalla dottrina dell'immagine sin dal Sabato santo 4 aprile 386, ascoltando un celebre discorso di S. Ambrogio che trattava appunto di questo argomento, in polemica col manicheismo: P. COURCELLE, Rechèrches sur les Confessions de saint Augustin, Paris 1950, p. 133 (cfr. G. BORTOLASO, Teologia dell 'immagine in Sant'Agostino e San Tommaso, in "La Civiltà Cattolica” 1967, III, 371 -380). 
Dio. Solo questa seconda è propriamente imago Dei, perché incorruttibile come Dio. Non vien meno neppure quando il corpo si corrompe, perché conosce Dio, lo invoca, lo ama, in altre parole è in comunione con lui (20).

Il corpo non è il luogo dell'immagine, tuttavia, oltre ad essere vestigium di Dio come tutto il creato, essendo fatto per il servizio dell'azione razionale, partecipa indirettamente alla qualità dell'immagine, sia perché esiste e vive, sia perché, capace di contemplare il cielo per la sua statura eretta, esso si avvicina all'immaginesomiglianza più del corpo animale.

Sulla natura del composto umano secondo Agostino, scrive il Trapè: "Circa l'unione di questi due elementi (= anima e corpo), contrariamente a quanto spesso si scrive, Agostino superò di molto lo spiritualismo di tipo ellenico, anche se continuò qua e là, specialmente nella predicazione, ad usarne il linguaggio. Non il corpo, ma il corpo corruttibile è carcere dell' anima (De civ. Dei 13, 16; In ps. 141, 18-19), la quale nata per informare il corpo, è ordinata per sua natura ad esso e senza di esso non può essere beata (De Gen. ad litt. 7, 27, 38). Ciò non toglie che egli insista sulla spiritualità e sulla immortalità personale dell'anima..." (21)

A conclusione possiamo dare una valutazione generale. La visione strettamente sintetica e globale della Bibbia sull'uomo incontra nei Padri una certa soluzione di continuità, a favore dello schema dualista, ossia di una più radicale distinzione degli elementi che "compongono" l'uomo. Occorre tuttavia dire che le posizioni dei Padri non sono uniformi ma differenziate, al punto che si potrebbe parlare di antropologie diverse: da quella che sembra più vicina alla concezione sintetica biblica (= scuola asiatica) a quella che collima spesso con il dualismo greco (= scuola alessandrina). Il maggior carattere "sintetico" della prima tendenza presenta una valutazione piena e positiva del corpo, o carne, in polemica con gli gnostici; la seconda tendenza si muove invece maggiormente all'ombra del pensiero platonico, per il quale il corpo e il sensibile sono realtà depotenziate. Ambedue le tendenze, comunque, si muovono nell'ambito della comune fede, dal momento che ambedue accolgono, pur con diverse sfumature, la fede nella creazione e nella redenzione di tutto l'uomo, come pure nella risurrezione: tre capisaldi che contraddistinguono il pensiero cristiano dal dualismo antropologico greco (22).

In una parola: il Kerigma cristiano, nato in contesto semitico, è stato inculturato nel vasto mondo greco-romano.

(20) Cfr, B. MONDIN, Antropologia teologia, Alba 1977, p 104.

(21) A. TRAPÈ, Patrologia, III, Torino 1978, p. 390. Un breve cenno a Gregorio M., il quale, commentando Gb 10, 8 "Manus tuae fecerunt me et plasmaverunt me totum in circuitu", sembra ispirarsi al commento di Ilario su Ps. 118, 73 e risale a Gen 1, 26 e 2, 7, orientando la sua riflessione nella stessa direzione di Ilano. Anche se in Gregorio. come del resto in Ilario, manca il supporto di un'antropologia di ordine filosofico o psicologico. Gregorio ammira la creazione dell'uomo: questi non è creato solo con la parola, ma è la risultante di una deliberazione ("consilium"); è plasmato dalIa terra con cura ("quasi per studium"), e riceve il soffio del Creatore che lo costituisce "spirito vivificante", per mostrare dalla dignità stessa dell'azione che lo pone in essere che egli è fatto a immagine del suo creatore. Comunque, la componente terrestre e carnale dell'uomo è il principio della sua debolezza Gregorio giunge a dire che la componente carnale rende l'uomo inferiore a se stesso (homo vero idcirco post culpam veniam meruit, quia per carnale corpus aliquid quo semletipso minor esset accepit) (Moralia in Iob IX, 50, 76; PL 75, 900 d - 901 a. Cfr. IX, 49, 75; PL 75, 900 a-c.) Nota il Solignac che Gregorio non spiega il senso di questa formula, ma con essa vuol sottolineare l'ambiguità della condizione umana e la dualità della natura (Solignac, col. 1423).

(22) Cfr S. RAPONI, Carne, in Dizionario di spiritualità dei laici, Milano 1981, pp. 94-95. 